\title{
A High Performance Glaucoma Screening Technique Using CNN Architecture
}

\author{
Srinivasa J, DeekshithaS,SushilKavin U, Dhiya N S, Chethan Kumar N S \\ Department of Electronics and communication, \\ C.Byregowda institute of technology, Kolar, India \\ srinivasa9397@gmail.com
}

\begin{abstract}
Glaucoma is a second irreversible eye disease across the world that can lead to blindness, if not treated early. At an early stage, it is very tedious to detect the disease as it does not show any symptoms. So, we build a supervised method for convolutional neural network to detect the disease as early as possible. In our model ACRIMA data set of 705 images are used where 396 are glaucomatous images and 309 are non-glaucomatous images. The complete data set is divided into $80 \%$ for training and $20 \%$ for testing. The data set is preprocessed before giving to the $\mathrm{CNN}$ model. Our CNN model consists of 8 layers where, 4 convolutional layers for feature extraction and 4 is fully connected layer to classify between glaucomatous and non-glaucomatous images. The best performance is obtained at the learning rate and epochs of 0.0001 and 100.In this work we evaluated the performance of Glaucoma-Deep system by plotting confusion matrix, the sensitivity, specificity, accuracy, precision (PRC) statistical measures and AUC of0.99
\end{abstract}

Keywords: CNN, ACRIMA ,AUC, Precision, Accu- racy ,Specifcity

\section{Introduction}

Glaucoma is considered to be the major common optic neuritis which is distinguished by continuous disappearance of retinal ganglion cells. Glaucoma is an optic disease with ephemeral heals which happens as the outcome of optic nerve harm due to which IOP built in the eye. The number of people affected by glaucoma across the world is approximated around 64 million in 2013,it'sgoingtoreach7̃6millionin2020andaround 111 million in 2040. In India, there are is an estimate of 12 million people affected by glaucoma, where the maximum number of people are not diagnosed. By 2020, in India it is expected to be 16 million [1]. Most of the affected peoples are inflammatory until it get to critical, and the number of specialists to analyze patients are insufficient [2]. A report shows that the damage of optic nerve fibers becomes noticeable and decreasing of visual field is detected when 40 percent of axonsarealreadylost. WHOhasproclaimedGlaucomato be 
the second big reason for vision loss across the world. The reduction in healthful neuron retinal tissues is been evaluated by the measurement of Cup to Disc Ratio (CDR).Typical rate of CDR for a good physical eye condition is 0.3. A Cup to Disc Ratio greater than 30 percent is contemplated to be troubled by Glaucoma. Although, the analytic identification by illustrating the CDR rates handcrafted for each image is work-exhaustive and also long running. Introduction of Computer-Aided Diagnosis systems is to have an alternative diagnosis to experts and to provide a costeffective solution to thepatients.As a result, the automated CAD screening systems have the proficiency to reduce time consumption and effort on the analyzing of glaucoma eye disease. According to various survey, scholars haverevealed that traditional classification's practice have difficulties of features extraction task that are not satisfying [3]. Extensive researches tried numerous image processing approaches to get over the before mentioned complication for a early recognition of Glaucoma. These days, Deep Neural Networks (DNNs) has been a vigorous experimentation, which can learnand extract large complicated characteristics from the input instinctively. Convolutional Neural Network architecture has been used victoriously in Computer Vision methods such as Classification and Semantic Segmentation. CNN are capable to learn the high complex features of the image without pre-processing and without correct assessment of geometric ONHstructures [4] [5].

\section{Definition and Theoretical Formation}

\subsection{Convolutional Neural Network}

It is a Deep neural network which is specialized for image recognition, where CNN work in the same way our cortex visualize works and recognize the image. The convolution layer takes the input as raw images and converts it to an array of pixels and it is given to the input of the Convolution neural network. Using the filter in the convolutional layer.The input image is executed by $2 \mathrm{D}$ convolution such that the output of this layer is know as feature map. The main use of CNN in our model is that where there is no any handcrafted feature extraction is needed. CNN is generally evolved under a cross entropy misfortune work that evaluates how approximately system boundaries concerning the ground-truth esteems in the preparation dataset [6]. Wecan partition the whole engineering into 2 expansive segment which is highlight learning and order area. The info picture is gone into a component extraction area where the removed component signal from the element extraction enters the grouping area. In the gathering region neural framework that works subordinate onthe part of the image and creates the image. [7] [8] The segment Extraction neural mastermind involves a part of convolutional layers and pooling layers sets. The convolutional layer as the name proposes changes over the picture using a convolution action. It might be thought of as the grouping of the modernized channels. The pooling layer unites the neighboring pixel into a singular pixel, along these lines the pooling layer diminishes the component of an image. As a basic concern of Convnet is the image, the movement of the convolution and the pooling layer are competently in a two- 
dimensional plane. This is one of the complexities betweenthe convent andotherneuralframeworks

In classification neural network, the fully connected layer operates on flatten in input where each input neuron is connected to all neurons.Toward the finish of the $\mathrm{CNN}$, the yield of the last Pooling Layer goes about as contribution to the Fully Connected Layer[9][10]. There can be at least one of these layers. Completely Connected layers perform arrangement dependent on the highlights removed by the past layers. Commonly, this layer is a conventional ANN containing a sigmoid initiation work, where yields are alikelihood.

\section{$3 \quad$ Methodology}

\subsection{CNNArchitecture}

A CNN Architecture consists of 8 layers - 4 Con- volutional layers and 4 Fully connected layers which is used for feature extraction and classification. CNN architectureismainlycomposedoftwo blocks.

Feature Extraction: The feature extraction block consistsoftheinputlayerfollowedbyfourconvolutional layers and max-pooling layers. The input layertakes the input as raw images and converts it to an array of pixels and it is given to the input of the convolutional layer. Using the filter in the convolutional layer it performs a $2 \mathrm{D}$ convolution on the input image to detect the presence of a set of features in the images and the output of this layer is called the feature map, the several convolution kernels are applied to the image and return the feature maps. We apply the filter to feature maps obtained with new kernels, which gives us new features maps to normalize and resize. Max pooling layers are used to decrease the dimension of the image which helps to reduce the parameters and increase the computational speed. An activation function called ReLU is used which is a non-linear function that increases the computational efficiency and avoids the vanishing gradient problem, additional to that batch normalization, dropout, Regularization (L2) techniques arealsousedtoavoidtheoverfitting.

\section{Classification:}

Classification block is also known asafullyconnectedlayer,thislayerisalwaysthelast layer of all neural networks. The classifier which we are using is Artificial neural network (ANN), the specialty of this network is that it connects every neurons of its layer's to the neuron of the next layer which yieldsbetter accuracy when compared to other classifiers. In our model we are using 4 fully connected layers, in the first 3 fully connected layers it works along with the dropout layer and the last fully connected layer works along with an activation (sigmoid) function which activate neurons within the range of 0 to 1 . We are also using anoptimizer called ADAM, to 
optimize the weight values during the backpropagation. The back-propagation process is nothing but it updates the weights of the model inreverseorder (from the last step to the first) based on the error rate calculated in the model using the optimizer. This back-propagation helps in increasing the accuracy of our model.

\section{The learning rate:}

The learning rate is scheduled in our model for tuning the parameter in an optimization algorithm which is used to determine the step size at each iteration while moving toward a minimum of a loss function. Epoch is defined before training our model, one epochswhen an entire dataset is passed in both backward and forword through the Artificial neural network at once. For our model epoch is given 100 times (100 epochs). Using all the extracted features the binary classifier classifies the images into infected and non-infected where 0 indicates glaucoma infected and 1 indicates noninfectedglaucoma.

\subsection{Dataset}

A new clinical ACRIMA database is consisting of 705 fundus images in which 396 are glaucomatous images and 309 are normal control images, with a resolution of $2048 \times 1536$. They are associated with the ACRIMA project and the fundus images were obtained from glaucomatous and normal patients. Most of the fundus images are focused on the optic disc. Topcon (TRC) and IMGEnet capture is a camera that is used to capture retinal funds images. In the field view of $35^{0}$ images were taken. The entire ACRIMA database image was checked out by two clinical experts.

\begin{tabular}{|l|l|l|l|}
\hline Layers & Layers name & Maps & kernal \\
\hline 1 & Image input & $224 \times 224 \times 3$ & - \\
\hline 2 & Convolutional 1 & $224 \times 224 \times 64$ & $5 \times 5$ \\
\hline 3 & ReLU & ReLU & - \\
\hline 4 & Maxpooling & $112 \times 112 \times 24$ & $2 \times 2$ \\
\hline 5 & Batch-normalization & batch-normalization & - \\
\hline 6 & Convolutional 2 & $112 \times 112 \times 64$ & $3 \times 3$ \\
\hline 7 & ReLU & ReLU & - \\
\hline 8 & Maxpooling & $56 \times 56 \times 64$ & $2 \times 2$ \\
\hline 9 & Batch-normalization & batch-normalization & - \\
\hline 10 & Dropout & $56 \times 56 \times 64$ & - \\
\hline 11 & Convolutional 3 & $56 \times 56 \times 64$ & $3 \times 3$ \\
\hline 12 & ReLU & ReLU & - \\
\hline 13 & Maxpooling & $28 \times 28 \times 64$ & $2 \times 2$ \\
\hline
\end{tabular}




\begin{tabular}{|l|l|l|l|}
\hline 14 & Batch-normalization & batch-normalization & - \\
\hline 15 & Convolutional 4 & $28 \times 28 \times 64$ & $3 \times 3$ \\
\hline 16 & ReLU & ReLU & - \\
\hline 17 & Maxpooling & $14 \times 14 \times 64$ & $2 \times 2$ \\
\hline 18 & Batch-normalization & batch-normalization & - \\
\hline 19 & Dropout & $14 \times 14 \times 64$ & - \\
\hline 20 & Fullyconnected & 4 & - \\
\hline 21 & Sigmoid & 1 & - \\
\hline 22 & Classification Output & binary crossentropy & - \\
\hline
\end{tabular}

\section{$4 \quad$ Result}

Our automatic glaucoma detecting model was built and tested on personal laptop with the processor of Intelcore i5 CPU@1.60 GHZ to $1.80 \mathrm{GHZ}$ and random access memory(RAM)of8GB.Thewasimplemented Using the 705 glaucoma and nonglaucoma images from ACRIMA data set our model was randomly trained and tested. Where, $80 \%$ of the images were randomly chosen for training and $20 \%$ of images were randomly chosen for testing our model. The model extracted the features and trained the model to classify images into glaucoma and non-glaucoma. The performance of our model was evaluated using statistical measures like precision, recall, f1score, accuracy, AUC-ROC curve for three different learning rates 0.1, 0.001, 0.0001 with 100 epochs for each learning rate. A comparison of three different learning rates with the statistical measures is shown in the table S2

TABLE 2: STATISTICAL MEASURE

\begin{tabular}{|c|c|c|c|c|}
\hline Learning rate & Accuracy & precision & specificity & Recall \\
\hline 0.1 & 0.84 & 0.89 & 0.61 & 0.81 \\
\hline 0.001 & 0.93 & 0.94 & 0.85 & 0.92 \\
\hline 0.0001 & 0.99 & 0.99 & 1 & 0.99 \\
\hline
\end{tabular}


Our model obtained best performance at 0.0001 learning rate for 100 epochs of accuracy 0.99 , f1- score of 0.99 , recall of 0.99 , precision of 0.99 and we also achieved the AUC-ROC curve area of $0.99 \mathrm{~s}$ shown in the Fig. 3

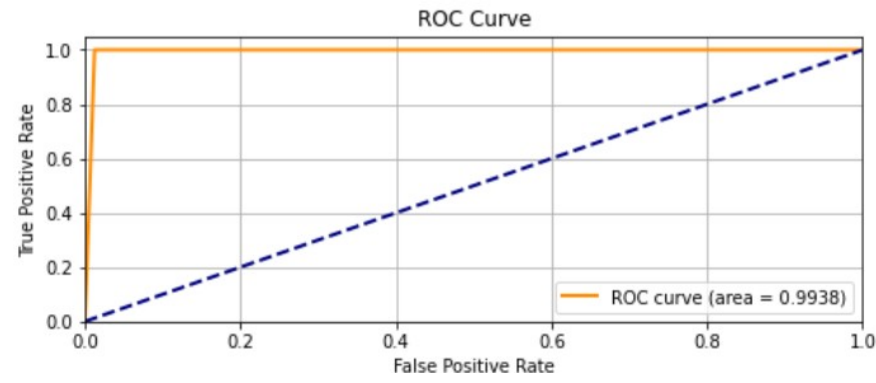

Fig. 3.AUC-ROCcurve

\section{Conclusion}

In our model, glaucoma can be diagnose using advance deep learning architecture using retinal funds images without using the hand-crafted features. To develop a Glaucoma deep learning algorithms, a convolutional neural network (CNN) supervised model is applied on 705 funds images to extract the features through multilayer from raw pixel intensities.As we examine the positive effect of architecture, the training strategy, the size of the Data set and collected data from the online clinical. We haveused data set of ACRIMA which is publicly available in online. In this implemented algorithm contains 4 convolution layer and 4 fully connected layer, we take on batch-normalization and max- pooling layer.

To increase our computation power and to avoid over-fitting we adopt dropout and dataare utilized to evaluateof glaucoma deep learning algorithm. On average, the PRC of $99 \%$, ACC of $99 \%$, SP of $99 \%$, SN of $99 \%$ were achieved. Conclusively, we evaluated the performance of the integration of the data from the clinical history with color fundus images. The result shows amendment in specificity and sensitivity with homogeneous AUCs. Further tests with more data and incipient architectural approaches should be developedandassessedtoattestthislineofwork 


\section{Reference}

1. G.Pavithra,T.Manjunath,D.Lamanietal.,"Detection ofsecondary glaucoma in human eyes using sophisticatedbio-medical image processing algorithms," in 2017 IEEE International Conference on Power, Control, Signals and InstrumentationEngineering.

2. S.Phan,S.Satoh,Y.Yoda,K.Kashiwagi,T.Oshika, J. O. I. R. R.Group et al., "Evaluation of deep convolutional neural networks forglaucomadetection,'Japanesejournalofophthalmology.

3. R.Touahri,N.Azizi,N.E.Benzebouchi,N.E.HAMMAMI, and O. Moumene, "A comparative study of convolutional neu- ral network and twin svm for automaticglaucomadiagno- sis"in2018International Conference on Signal, Image, Vision andtheirApplications(SIVA).IEEE,2018,pp.1-5.

4. A.Diaz-Pinto,S.Morales,V.Naranjo,T.Köhler, J.M.Mossi,andA.Navea, "Cnnsfor automatic glaucoma assessment using fundus images: an extensive validation," Biomedicalengineeringonline,vol.18,no.1,p.29,2019.

5. A. Serener and S. Serte, "Transfer learning forearlyandadvancedglaucomadetectionwithconvolutional- neuralnetworks,"in2019 Medical Technologies Congress (TIPTEKNO). IEEE, 2019, pp.1-4.

6. S. T. Ahmed, "A study on multi objective optimal clustering techniques for medical datasets," 2017 International Conference on Intelligent Computing and Control Systems (ICICCS), Madurai, 2017, pp. 174-177, doi: 10.1109/ICCONS.2017.8250704.

7. K. D. Singh and S. T. Ahmed, "Systematic Linear Word String Recognition and Evaluation Technique," 2020 International Conference on Communication and Signal Processing (ICCSP), Chennai, India, 2020, pp. 0545-0548, doi: 10.1109/ICCSP48568.2020.9182044

8. S. T. Ahmed, H. K. Priyanka, S. Attar and A. Patted, "Cataract density ratio analysis under color image processing approach," 2017 International Conference on Intelligent Computing and Control Systems (ICICCS), Madurai, 2017, pp. 178-180, doi: 10.1109/ICCONS.2017.8250705.

9. Gunashree, M., Ahmed, S. T., Sindhuja, M., Bhumika, P., Anusha, B., \&Ishwarya, B. (2020). A New Approach of Multilevel Unsupervised Clustering for Detecting Replication Level in Large Image Set. Procedia Computer Science, 171, 1624-1633. https://doi.org/10.1016/j.procs.2020.04.174

10. Dafni Rose J, Vijayakumar K, "Data Transmission Using Multiple Medium Concurrently", IJET,2018.

11. K. Vijayakumar, S. Suchitra and P. Swathi Shri, "A secured cloud storage auditing with empirical outsourcing of key updates", Int. J. Reasoning-based Intelligent Systems, Vol. 11, No. 2, 2019. 